\title{
AN EXCESSIVE GAG REFLEX TREATED BY HYPNOSIS A Case Report
}

\author{
LIEUTENANT-COLONEL L. J. KESSEL, B.D.S., F.D.S., R.C.S.(Eng.), R.A.D.C. \\ LIEUTENANT-COLONEL R. J. BELAS, T.D., M.B., M.R.C.Psych., D.P.M., \\ R.A.M.C.
}

\section{British Military Hospital, Hong Kong}

M.B., a 46 year old housewife, was referred to the dental department for advice and possible treatment for a sensitive gag reflex to her upper denture.

The denture, which was horse-shoe shaped in design, had been fitted four weeks earlier with the posterior edge well clear of the soft palate, but the patient had been unable to tolerate it for more than five minutes at a time, before she commenced gagging. An upper denture had first been constructed in 1970 after the upper posterior teeth had been removed, but owing to the persistent gagging, she had been unable to tolerate it. The patient had been without an upper prosthesis until she decided to have her present set constructed to offset the "sagging" effect in her upper cheeks due to the lack of dental support.

On examination the horse-shoe shaped denture fitted well, the retention was good, and the occlusion normal. However, shortly after its insertion gagging commenced. As the patient seemed a sensible, emotionally stable individual it was decided to refer her for psychiatric evaluation to see if hypnosis could overcome her problem.

Psychiatric assessment confirmed her to be a good personality and emotionally stable. She gave no psychiatric history in herself or her family. As she appeared to be enthusiastic about the use of hypnosis in an attempt to overcome her problem this form of therapy was embarked upon.

The treatment regime employed was as follows: The patient was asked to lie down on a comfortable bed, to concentrate her gaze on the tip of a pen held in front of and just above her eyes and a simple form of relaxation therapy was embarked upon, starting at the feet and working upwards to her head. A light hypnotic trance was thus achieved. At the second session two days later she was again hypnotised and it was suggested that the wearing of her dentures would become more comfortable: Four days later when she attended for the third session she stated that she had been able to wear the denture without discomfort since the previous session. On this occasion her trance state was deepened and it was suggested that the wearing of the denture would remain comfortable, permanently. Following this a post hypnotic suggestion was given that in future three taps with the pen by the therapist would be effective in inducing a trance. This was done to speed up later sessions and to test the depth of trance.

Altogether six sessions were utilised for treatment, the latter four for reenforcement purposes, and the last one also for removing the post hypnotic suggestion regarding tapping the pen. 
It was also strongly suggested to the patient at each session that she would never allow herself to be hypnotised unless it be for qualified medical or dental purposes. Three months later she was reviewed and had remained sympton free.

\section{Discussion}

Gagging during dental procedures is not uncommon, frequently occurring during impression taking, intra oral radiography and insertion of upper dentures.

Patients with a sensitive gag reflex during impression and intra oral radiography can frequently be 'talked' through the procedure. In those cases which are resistant to this approach one can resort to topical anaesthesia such as sucking a benzocaine lozenge or even injecting local anaesthetic beforehand. Often patients who gag during impression taking, surprisingly have no problems when the dentures are finally inserted.

Initial gagging on insertion of a new upper denture can often be overcome by sucking a sweet. However there are a few cases, such as this described here, where one has to resort to a horse-shoe shaped design in the upper denture to keep the posterior edge clear of the soft palate. In spite of this there are still those cases where all conventional methods fail and where hypnosis could be successful:

The use of hypnosis in the treatment of excessive gag reflex is not new and has already been reported (Dorcus 1965, Shaw 1958, Sector 1961 and others).

The method used in this case is so simple and safe that it is suggested that it could be used more frequently with success and benefit to carefully selected patients.

\section{REFERENCES}

DORCUS, R. M. (1956). Hypnosis and its Therapeutic Applications. 1st ed McGraw-Hill Book Co. Toronto.

SECTOR, I. I. (1960). Amer. J. Clin. Hypnos. January edition.

SHAW, S. I. (1958): Clinical Applications of Hypnosis in Dentistry. 1st ed. W. B. Saunders Co. Philadelphia. 